\title{
MANFREDA LITTORALIS (AGAVACEAE), NUEVA ESPECIE DE GUERRERO Y OAXACA, MÉXICO
}

\author{
Abisaí García-Mendoza \\ Alejandro Castañeda Rojas \\ E \\ I. Sonia Franco Martínez \\ Jardín Botánico \\ Instituto de Biología, UNAM \\ Apartado postal 70-614 \\ 04510 México, D.F. \\ email: abisai@mail.ibiologia.unam.mx
}

\section{RESUMEN}

Se describe e ilustra Manfreda littoralis, de la costa del Oceáno Pacífico en los estados de Guerrero y Oaxaca. La nueva especie pertenece al grupo de Manfreda guttata (Jacobi et Bouché) Rose y muestra similitudes con M. guttata (Jacobi et Bouché) Rose y M. planifolia (S. Watson) Rose.

\section{ABSTRACT}

Manfreda littoralis, a new species from the seashores of Guerrero and Oaxaca is described and illustrated. It belongs to the Manfreda guttata (Jacobi et Bouché) Rose group, and is similar to M. guttata (Jacobi et Bouché) Rose and M. planifolia (S. Watson) Rose.

El género Manfreda, de distribución principalmente norteamericana, fue revisado taxonómicamente por Verhoek-W. (1975), quien menciona 19 especies, las cuales crecen de West Virginia en los Estados Unidos, hasta Honduras y El Salvador en Centroamérica. México es el centro de riqueza y diversidad del género, ya que de las 28 especies identificadas en la actualidad, 27 (96\%) crecen en el país (Espejo \& López-F., 1993; GarcíaMendoza y Galván, 1995). Este número seguramente se verá incrementado, ya que existen plantas que no se circunscriben a las especies publicadas en los trabajos de Verhoek-W. (1975) y Piña Luján $(1985,1985 a, 1985 b, 1986)$. El taxon aquí descrito es uno de ellos, ubicado anteriormente por Verhoek-W. (1975:316) en sus especies inciertas del grupo de M. guttata.

Manfreda littoralis García-Mend., A. Castañeda et S. Franco, sp. nov. Fig. 1.

Planta herbacea, rhizomate conico-truncato, stolonifero, vestigiis basalibus foliorum membranaceis. Folia 9-20(-27) cm longa, 2-5(-6) cm lata, elliptica vel lanceolato-elliptica, 
semisucculenta, viridi-lutea, maculis purpureis vel atroviridibus. Bracteae inferiores inflorescentiae foliiformes. Flores $1.5-2(-2.5) \mathrm{cm}$ longi, odore acro, nectare abundanti, perianthii tubo 1-2(-2.8) mm longo, segmentis oblongis, 9-10(-11) mm longis, 1.5-2(-3.5) mm latis, erectis vel reflexis, ovario $3-5(-6) \mathrm{mm}$ longo, 1.5-2.5(-4) $\mathrm{mm}$ lato, in tubo florali minime penetranti. Fructus $0.9-1 \mathrm{~cm}$ longus, $0.8-1 \mathrm{~cm}$ diametro, subglobosus, humeri signo leniter manifesto.

Planta con rizoma erecto, $3-5 \mathrm{~cm}$ de alto, $2.5-3 \mathrm{~cm}$ de diámetro en la base, cónicotruncado, estolonífero; restos basales de las hojas 1.5-2.5(-3.5) cm de largo, membranáceos; raíces carnosas y fibrosas. Hojas 4 a 6 , de $9-20(-27) \mathrm{cm}$ de largo, 2-5(-6) cm de ancho, elípticas a elíptico-lanceoladas, angostándose en un pseudopecíolo envainante y acanalado de $0.5-1 \mathrm{~cm}$ de ancho, semisuculentas, planas, postradas o semierectas, verde-amarillentas con máculas purpúreas o verdes oscuras, glabras, margen entero al tacto, al microscopio finamente papiloso en algunas secciones, sobre una banda hialina angosta, ápice apiculado o acuminado, punto corto. Inflorescencia de $60-70(-80) \mathrm{cm}$ de alto, erecta a ligeramente arqueada, porción floral de 3.5-4.5(-6.5) cm largo, densa, con 11-14(-24) flores; brácteas del pedúnculo 9-12(-15), con la base envainante, las inferiores foliiformes, las superiores bracteiformes, la segunda bráctea basal de 10-18(-24) cm de largo, 1-2(-4) cm de ancho, verde-amarillenta; bractéolas florales iguales en longitud o más largas que el ovario, pedúnculo de la inflorescencia rojizo en la base, verdoso a glauco en la porción superior. Flores de 1.5-2(-2.5) cm de largo, erectas, sésiles, en ocasiones las inferiores sobre pedicelos menores de $1 \mathrm{~mm}$, verde-amarillentas, de olor acre, con néctar abundante; ovario de 3-5(-6) mm de largo, 1.5-2.5(-4) $\mathrm{mm}$ de ancho, prolongándose menos de $1 \mathrm{~mm}$ en el tubo, cilíndrico, verde-amarillento; tubo del perianto de 1-2(-2.8) $\mathrm{mm}$ de largo, $2-3 \mathrm{~mm}$ de diámetro, unido al ovario en ángulo recto y sin constricción, segmentos de 9-10(-11) mm de largo, 1.5-2(-3.5) mm de ancho, oblongos, erectos o reflexos, ápice semisuculento, cuculado; los filamentos exceden al tubo por (1-)1.4-1.8 cm, adnados al tubo en toda su longitud, libres en la base de los tépalos, dispuestos en un solo plano, rectos en la antesis, blanquecinos; anteras de 7-8(-9) $\mathrm{mm}$ de largo, 1-1.5 mm de ancho, blanquecinas; el estilo excede al tubo por $1.6-2 \mathrm{~cm}$; estigma trilobado. Cápsulas de $0.9-1 \mathrm{~cm}$ de largo, $0.8-1 \mathrm{~cm}$ de diámetro, subglobosas, triquetras, con los restos del perianto persistentes, marca del hombro (ápice redondeado de la cápsula) poco evidente. Semillas de 3-4 mm de largo, $3 \mathrm{~mm}$ de ancho, plano-convexas, negruzcas, opacas.

TIPO: México, Guerrero, municipio de Acapulco, parque nacional El Veladero, barranca al $\mathrm{N}$ de la colonia Independencia, $220 \mathrm{~m}$, selva mediana subcaducifolia, 14 agosto 1999, A. García-Mendoza, A. Castañeda y S. Franco 6900 (Holotipo: MEXU; isotipo: MO).

Paratipos: México, Guerrero, Pie de la Cuesta, about 10 miles $\mathrm{N}$ of Acapulco, L. H. McDaniels 174 (BH, F); municipio de Acapulco, parque nacional El Veladero, $N$. Noriega 685 (FCME); Oaxaca, distrito de Pochutla, municipio de Santa María Huatulco, al NO de Playa Cacaluta, G. Castillo et al. 9358 (MEXU, XAL); distrito de Pochutla, municipio de Santa María Huatulco, al NO de la Bahía Santa Cruz, G. Castillo et al. 9443 (MEXU, XAL); distrito de Tehuantepec, municipio de Santiago Astata, Río Zimatán, E. Martínez et al. 32447, 32524 (MEXU). 


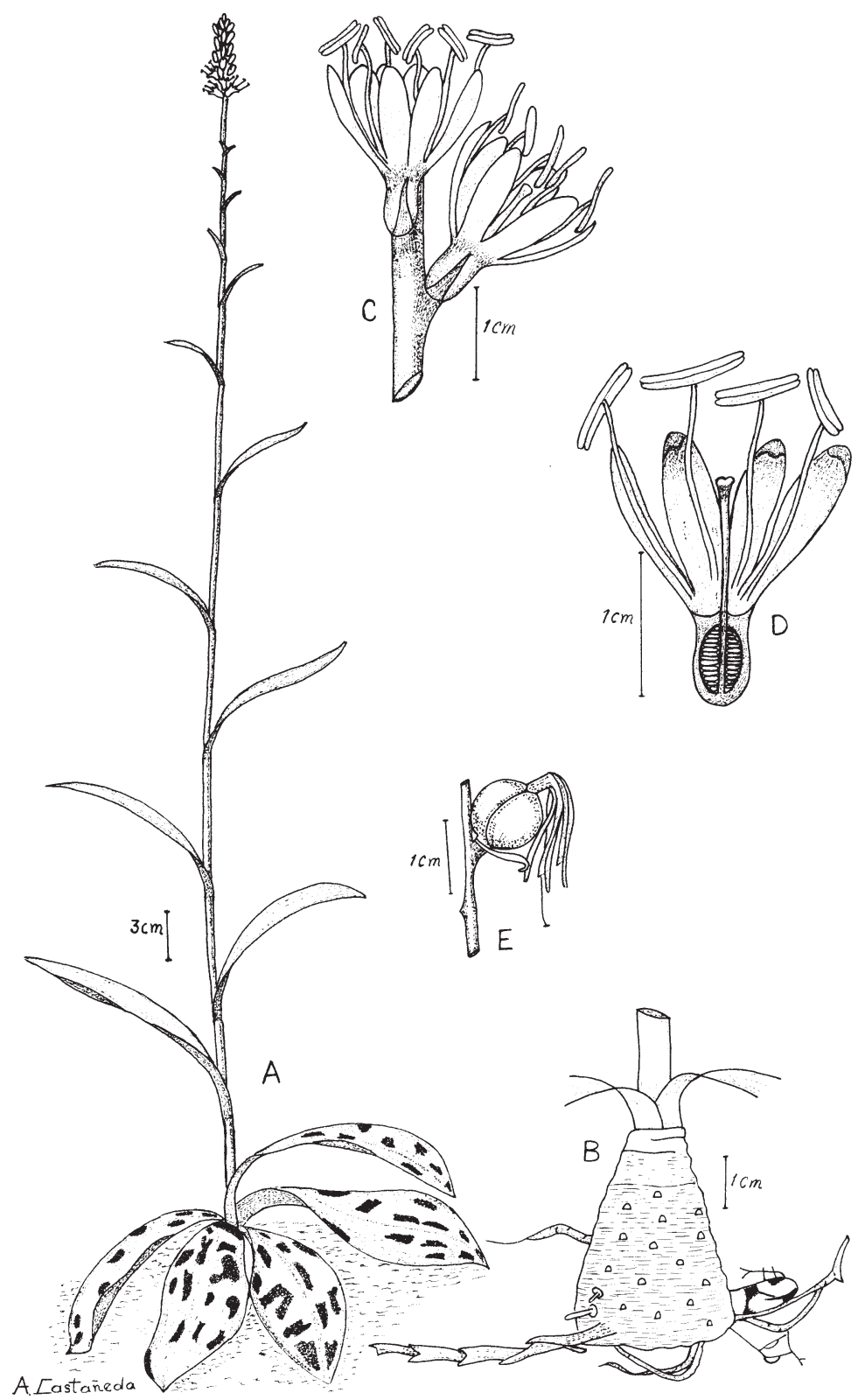

Fig. 1. Manfreda littoralis. A. planta con inflorescencia; B. rizoma estolonífero; C. flores; D. corte longitudinal de la flor; E. fruto. Basado en los ejemplares García-Mendoza et al. 6900 y G. Castillo et al. 9443. Ilustrado por Alejandro Castañeda Rojas. 
Distribución y hábitat: Manfreda littoralis crece en sitios rocosos, donde se acumulan pequeñas cantidades de suelo rico en materia orgánica y de textura arenosa, dentro de la selva mediana subcaducifolia y selva baja caducifolia con Bursera, Euphorbia, Ficus, Genipa, Lonchocarpus, Muntingia y Sterculia, en altitudes de 20 a $260 \mathrm{~m}$. Florece de fines de agosto a principios de octubre y fructifica en noviembre y diciembre. Es una especie aparentemente escasa en la naturaleza y endémica de la vegetación costera en los estados de Guerrero y Oaxaca. Su nombre alude a la zona cercana al mar, donde se le encuentra.

Discusión: Manfreda littoralis pertenece al grupo de $M$. guttata, reconocido por Verhoek-W. (1975) en función de sus características florales y foliares. En el conjunto, las hojas son delgadas a semisuculentas, con el margen foliar hialino, liso o áspero al tacto y papiloso o eroso-denticulado al microscopio, el ovario de las flores se proyecta ligeramente dentro del tubo de la flor, lo que provoca que el perianto persista en las cápsulas y deje una cicatriz en forma de anillo alrededor del "hombro" (parte apical redondeada de la cápsula). Las especies reunidas por Verhoek-W. (1975) en este grupo son: Manfreda guttata (Jacobi et Bouché) Rose, M. planifolia (S. Watson) Rose, M. pringlei Rose y M. rubescens Rose, todas endémicas de México. Posteriormente fue descrita M. fusca Ravenna (1987) con material procedente de Guatemala, sin ser adjudicada a conjunto alguno; sin embargo, al estudiar los ejemplares de esta especie depositados en MEXU, pudimos asignarla sin problemas al grupo guttata.

Manfreda littoralis se distingue por su rizoma cónico-truncado, estolonífero, por los restos basales de las hojas membranáceos, por sus hojas elípticas o elíptico-lanceoladas, verde-amarillentas con máculas purpúreas o verdes oscuras, parte fértil de la inflorescencia corta y por el tamaño pequeño de las flores con el tubo periantal abreviado y el ovario que se proyecta muy poco dentro del tubo. Es característico también el olor acre de las flores, el abundante néctar que producen, sobre todo en las mañanas y el tipo de hábitat donde se encuentra. Verhoek-W. (1975) señala que $M$. guttata y $M$. pringlei se propagan vegetativamente mediante rizomas estoloníferos que dan origen a nuevas plantas. Esta característica es exclusiva del grupo guttata y se presenta también en $M$. littoralis. Cabe agregar que la presencia de un aroma a cebolla se menciona para las flores de M. guttata, mientras que Ravenna (1987) refiere un olor fétido para las mismas en $M$. fusca.

Dentro del grupo, las especies más parecidas a $M$. littoralis son $M$. planifolia y $M$. guttata, de las que se separa por las características mostradas en el Cuadro 1.

\section{CLAVE PARA LOS TAXA DEL GRUPO DE MANFREDA GUTTATA}

1 Hojas elípticas, elíptico-lanceoladas u oblongas.

2 Flores 1.5-2(-2.5) cm de largo; tubo 1-2(-2.8) mm de largo; los filamentos exceden al tubo por (1-)1.4-1.8 cm, adnados en toda la longitud del tubo

M. littoralis

2 Flores 3.2-4 cm de largo; tubo 5-7 mm de largo; los filamentos exceden al tubo por 2-5.2 cm, insertos a la mitad del tubo M. planifolia

1 Hojas lineares, linear-lanceoladas a lanceoladas. 
3 Filamentos adnados en toda la longitud del tubo.

4 Hojas menos de $1 \mathrm{~cm}$ de ancho, margen eroso-denticulado; cápsulas 1.6-2.4 cm largo, $1-1.9 \mathrm{~cm}$ diámetro, elipsoideas

M. rubescens

4 Hojas más de $1 \mathrm{~cm}$ de ancho, margen liso o papilado; cápsulas $1.5 \mathrm{~cm}$ largo, 1.5 $\mathrm{cm}$ diámetro, globosas M. guttata

3 Filamentos insertos a la mitad del tubo.

5 Tubo floral 0.9-1 cm largo, ovario 1.2-1.7 cm largo; especie conocida sólo de las montañas del centro de Guatemala M. fusca

5 Tubo floral 1-1.5(-1.7) cm largo, ovario 1-1.2 cm largo; especie distribuida en el Eje Neovolcánico y montañas de Oaxaca, México M. pringlei

Cuadro 1. Características comparativas de Manfreda littoralis y especies afines. Los datos de M. guttata están tomados de Verhoek-W. (1975) y de observaciones propias; los de M. planifolia son de Watson (1887) y Verhoek-W. (1975).

\begin{tabular}{|c|c|c|c|}
\hline & M. littoralis & M. guttata & M. planifolia \\
\hline Rizoma & $\begin{array}{l}3-5 \times 2.5 \mathrm{~cm} \text {, cónico- } \\
\text { truncado, estolonífero }\end{array}$ & $\begin{array}{l}1.8-4 \times 1.3-3 \mathrm{~cm} \text {, globo } \\
\text { so, estolonífero }\end{array}$ & $4 \times 4 \mathrm{~cm}$, globoso \\
\hline Restos foliares & $\begin{array}{l}1.5-2.5(-3.5) \mathrm{cm} \text { de lar- } \\
\text { go, membranáceos }\end{array}$ & $\begin{array}{l}3-6 \mathrm{~cm} \text { de largo, fibro- } \\
\text { sos }\end{array}$ & $\begin{array}{l}3.8-5 \mathrm{~cm} \text { de largo, fibro } \\
\text { sos }\end{array}$ \\
\hline Número de hojas & $4-6$ & $2-7$ & $4-5$ \\
\hline Hojas & $\begin{array}{l}9-20(-27) \times 2-5(-6) \mathrm{cm} \text {, } \\
\text { elípticas a elíptico-lan- } \\
\text { ceoladas, pseudopecio- } \\
\text { ladas }\end{array}$ & $\begin{array}{l}(8-) 14-38 \times 1.3-3.1 \mathrm{~cm}, \\
\text { linear-lanceoladas a lan } \\
\text { ceoladas, angostas ha- } \\
\text { cia la base }\end{array}$ & $\begin{array}{l}21-30 \times(2.5-) 3.1-6.1 \mathrm{~cm} \text {, } \\
\text { elípticas a oblongas, } \\
\text { pseudopecioladas }\end{array}$ \\
\hline $\begin{array}{l}\text { Margen de la hoja } \\
\text { (al microscopio) }\end{array}$ & finamente papiloso & eroso-denticulado & finamente denticulado \\
\hline Color de las hojas & $\begin{array}{l}\text { verde-amarillentas con } \\
\text { máculas purpúreas o } \\
\text { verdes oscuras }\end{array}$ & $\begin{array}{l}\text { glaucas a verdes páli- } \\
\text { das, a veces moteadas } \\
\text { de púrpura }\end{array}$ & verdes?, sin máculas \\
\hline $\begin{array}{l}\text { Largo de la inflores- } \\
\text { cencia }\end{array}$ & $60-70(-80) \mathrm{cm}$ & (61-)90-156 cm & $120-152 \mathrm{~cm}$ \\
\hline $\begin{array}{l}\text { Largo de la porción } \\
\text { floral de la inflorescen } \\
\text { cia }\end{array}$ & $3.5-4.5(-6.5) \mathrm{cm}$ & $(2.3-) 8-14 \mathrm{~cm}$ & $11 \mathrm{~cm}$ en cultivo \\
\hline Flores & $\begin{array}{l}1.5-2(-2.5) \mathrm{cm} \text {, verde- } \\
\text { amarillentas, glaucas, } \\
\text { olor acre }\end{array}$ & $\begin{array}{l}2-3.9 \mathrm{~cm} \text {, verde-amari- } \\
\text { llentas con tintes pur- } \\
\text { púreos, olor a cebolla }\end{array}$ & $3.2-4 \mathrm{~cm}$ \\
\hline
\end{tabular}


Cuadro 1. Continuación.

\begin{tabular}{|c|c|c|c|}
\hline & M. littoralis & M. guttata & M. planifolia \\
\hline Ovario & $3-5(-6) \times 1.5-2.5(-4) \mathrm{mm}$ & $6-12(-15) \times 3-4 \mathrm{~mm}$ & $15 \times 6-7 \mathrm{~mm}$ en cultivo \\
\hline Tubo del perianto & $1-2(-2.8) \times 2-3 \mathrm{~mm}$ & 3-12 mm & $5-7 \mathrm{~mm}$ \\
\hline $\begin{array}{l}\text { Segmentos del perian } \\
\text { to }\end{array}$ & $\begin{array}{l}9-10(-11) \times 1.5-2(-3.5) \\
\mathrm{mm}\end{array}$ & $(6-) 10-15 \times 2-3 \mathrm{~mm}$ & $12-19 \times 4 \mathrm{~mm}$ \\
\hline Filamentos & $\begin{array}{l}(1-) 1.4-1.8 \mathrm{~cm} \text { de largo, } \\
\text { adnados al tubo }\end{array}$ & $\begin{array}{l}2-2.9(-4.1) \mathrm{cm} \text { de largo, } \\
\text { adnados al tubo }\end{array}$ & $\begin{array}{l}2.1-5.2 \mathrm{~cm} \text { de largo, in } \underline{n} \\
\text { sertos a la mitad del tu} \underline{u} \\
\text { bo }\end{array}$ \\
\hline Fruto & $\begin{array}{l}0.9-1 \times 0.8-1 \mathrm{~cm} \text {, sub- } \\
\text { globoso, marca del hom } \\
\text { bro poco evidente }\end{array}$ & $\begin{array}{l}1.6-2.4 \times 1-1.9 \mathrm{~cm} \text {, elip } \\
\text { soideo, marca del hom } \\
\text { bro } 2-3 \mathrm{~mm}\end{array}$ & $\begin{array}{l}1.8 \mathrm{~cm} \text {, ovado, con mar } \\
\text { ca del hombro }\end{array}$ \\
\hline Hábitat & $\begin{array}{l}\text { selva mediana subcadu } \\
\text { cifolia y baja caducifolia } \\
\text { alt. } 20-260 \mathrm{~m}\end{array}$ & $\begin{array}{l}\text { pastizales, sitios abier- } \\
\text { tos o rocosos } \\
\text { alt. } 1800-2400 \mathrm{~m}\end{array}$ & encinares \\
\hline Distribución & Gro., Oax. & $\begin{array}{l}\text { Ags., Chih., Dgo., Jal., } \\
\text { Qro., S.L.P., Zac. }\end{array}$ & Chih., Son. \\
\hline
\end{tabular}

\section{AGRADECIMIENTOS}

Agradecemos al Dr. Fernando Chiang la revisión del manuscrito y la elaboración de la descripción en latín. A la Dra. Raquel Galván V. y a dos revisores anónimos los comentarios que enriquecieron este trabajo. Al Biól. Esteban Martínez Salas por su entusiasmo en la colecta de esta especie. A los curadores de los herbarios FCME, MEXU y XAL por sus amables atenciones.

\section{LITERATURA CITADA}

Espejo, A. y A.R. López-Ferrari. 1993. Manfreda, en: Las monocotiledóneas mexicanas, una sinopsis florística. Consejo Nacional de la Flora de México, Universidad Autónoma Metropolitana Unidad Iztapala, Comisión Nacional de la Biodiversidad. México, D.F. pp. 33-36.

García-Mendoza, A. y R. Galván V. 1995. Riqueza de las familias Agavaceae y Nolinaceae en México. Bol. Soc. Bot. México 56: 7-24.

Piña Luján, I. 1985. Consideraciones sobre el género Manfreda I. Cact. Suc. Mex. 30(2): 27-32.

Piña Luján, I. 1985a. Consideraciones sobre el género Manfreda II. Cact. Suc. Mex. 30(3): 56-64.

Piña Luján, I. 1985b. Consideraciones sobre el género Manfreda III. Cact. Suc. Mex. 30(4): 84-90.

Piña Luján, I. 1986. Consideraciones sobre el género Manfreda IV. Cact. Suc. Mex. 31(1): 12-18. 
Ravenna, P. 1987. Notes on Agavaceae I. A new species of Manfreda. Herbertia 43(1): 17-19.

Verhoek-W., S. E. 1975. A study of the tribe Poliantheae (including Manfreda) and revisions of Manfreda and Prochnyanthes (Agavaceae). Ph D. Thesis. Cornell University, Ithaca, Nueva York. 403 $\mathrm{pp}$.

Watson, S. 1887. Contributions to American botany. 2. Descriptions of some new species of plants. Proc. Amer. Acad. Arts 22: 466-481.

Recibido en enero de 2000. Aceptado en marzo de 2000. 